Annuaire suisse de politique de développement

$17 \mid 1998$

Propriété intellectuelle : quels enjeux pour les pays en développement ?

\title{
4. Environnement et développement
}

\section{CpenEdition}

\section{Journals}

Édition électronique

URL : http://journals.openedition.org/aspd/771

DOI : 10.4000/aspd. 771

ISSN : 1663-9669

Éditeur

Institut de hautes études internationales et du développement

Édition imprimée

Date de publication : 1 avril 1998

Pagination : 191-213

ISSN : 1660-5934

Référence électronique

《4. Environnement et développement », Annuaire suisse de politique de développement [En ligne], 17|

1998, mis en ligne le 31 juillet 2012, consulté le 08 septembre 2020. URL : http://

journals.openedition.org/aspd/771; DOI : https://doi.org/10.4000/aspd.771

(c) The Graduate Institute I Geneva 


\section{ENVIRONNEMENT ET DÉVELOPPEMENT}

L A CONFÉRENCE des Nations Unies sur l'environnement et le développement (CNUED), qui s'est tenue à Rio en 1992, était une étape importante sur la voie du développement durable. Le fait déterminant est la prise de conscience des chefs d'Etat et de gouvernement du monde entier, des leaders de l'économie et des représentants des différents groupes sociaux, de la nécessité d'une nouvelle façon de penser et d'agir. Le concept de développement durable tend à (ré) concilier la solidarité sociale, la responsabilité écologique et l'efficacité économique. Depuis le Sommet de Rio des efforts et initiatives ont été entrepris à l'échelon international, national et local pour concrétiser le développement durable.

En Suisse, la Confédération a préparé deux documents consacrés à la mise en œuvre du développement durable sur le plan national, qui ont été présentés lors de l'Assemblée extraordinaire des Nations Unies (appelée également UNGASS ou Rio + 5) qui s'est tenue à New York du 23 au 27 juin 1997. Le premier établit un état des réalisations; le second, intitulé «Stratégie», évoque les mesures complémentaires visant à mettre en æuvre la politique de développement durable. La séance extraordinaire de l'ONU avait pour objectif d'établir le bilan des réalisations et de fixer les priorités pour les années à venir. Ce processus d'évaluations périodiques est important et unique dans le suivi des conférences internationales. De l'avis général, les résultats du Sommet Rio +5 ont été très décevants. La Suisse a également participé activement aux Conférences des parties des conventions qui avaient été signées à Rio et ratifiées par le Parlement (climat et biodiversité).

Les organisations non gouvernementales d'environnement et de développement suisses ont conduit durant l'année 1997 des campagnes d'information visant à la concrétisation du développement durable.

\subsection{CINQ ANS APRÈS LE SOMMET DE LA TERRE}

\section{$\square$ Suivi de Rio en Suisse et préparation au Sommet Rio +5}

Lors de la Conférence des Nations Unies sur l'environnement et le développement en juin 1992, la communauté internationale a établi un programme politique et institutionnel qui doit servir de référence au cours des prochaines années pour lutter contre les problèmes environnementaux globaux et atteindre le développement durable (Agenda 21).

En Suisse, le Sommet de la Terre a introduit une réorientation de la politique de l'environnement. D'une politique fortement axée sur les prescriptions et les interdictions, complétée dans certains domaines par un système de subventions et de contributions, on est passé à une politique de l'environnement, ces dernières années, qui a eu un recours accru aux instruments économiques et a 
mieux intégré l'environnement dans d'autres domaines politiques'. La mise en œuvre d'un Agenda 21 au niveau suisse participe à la réorientation de la politique de l'environnement, ainsi qu'à l'internalisation et la globalisation de ces mesures.

Depuis la CNUED, la Confédération a rédigé un certain nombre de rapports et a créé une double structure institutionnelle; interne à l'administration (le CIRio), et externe en tant qu'elle intègre des milieux extérieurs: un groupe de liaison et sept groupes de travail. Cette structure avait pour objectif de mieux coordonner le travail et de faciliter les échanges d'informations. (Voir plus loin l'encadré «Processus de Rio 1992-1997».)

En avril 1997, quatre ans après la mise en place de cette structure, le Conseil fédéral a reconduit la structure du CIRio. Par contre, le groupe de liaison a été dissous et sera remplacé par un Conseil pour le développement durable (voir cidessous). Ont également été dissous trois des groupes de travail (transferts de technologies et coopération technique, incitation et internalisation des externalités, cohérence de la législation et subventions). Seul un rapport «Transfert de technologies» a été publié ${ }^{2}$, mais de l'avis même d'un des membres du groupe, Andràs November, «le rapport n'a jamais obtenu l'attention qu'il méritait (...). L'administration fédérale n'était pas trop pressée de diffuser une étude, qui en définissant une politique, pouvait aller à l'encontre des vues des divers offices fédéraux et révéler leur divergence en matière de politique de transfert de technologies de l'environnement ${ }^{3}$. Quatre groupes de travail continuent leurs rencontres: il s'agit des groupes climat, biodiversité et forêt, ainsi que du Forum commerce et environnement.

\section{«Le développement durable en Suisse - Stratégie», du Conseil fédéral}

Le 28 février 1996, le Conseil fédéral a pris connaissance de la première version du rapport du CIRio intitulé Le développement durable en Suisse. A cette occasion il avait donné mandat au CIRio d'élaborer un plan d'action suisse pour le développement durable. Cette tâche a été confiée à sept personnalités, réunies dans un groupe de travail intitulé «Conseil pour le développement durable» ${ }^{4}$. Ce groupe a présenté son rapport, intitulé Le développement durable - Plan d'action pour la Suisse, en janvier 1997. Sur la base de ce rapport, le CIRio a ensuite préparé la stratégie Le développement durable en Suisse.

Deux documents parus en avril 1997 servent de base à l'évaluation et au plan d'action pour une Suisse dirigée selon les principes du développement durable:

- Le développement durable en Suisse - Etat des réalisations, rapport du CIRio, mis à jour en avril 1997;

- Le développement durable en Suisse - Stratégie, du Conseil fédéral.

Ces deux documents sont complémentaires. Le document Etat des réalisations explique le concept de développement durable, pose les exigences à remplir et définit les principales stratégies à mettre en place en Suisse. Le rapport tend à

1. Philippe Roch, in La Vie économique, «Politique de l'environnement, une nouvelle dimension après Rio?», 12/1996.

2. CIRio, Transfer and Cooperation in the Area of Environmentally Sound Technologies, final report of the IDC Rio Working Group «Technology Transfer/Cooperation», Bern, September 1995.

3. Annuaire Suisse-Tiers Monde 1997, p. 253.

4. Le Conseil pour le développement durable était présidé par Ernst Blaser et composé de six autres personnalités: Fritz Fahrni, Jacques Forster, Charles Kleiber, Jakob Nüesch, Anne Petitpierre, Roman Rudel. 


\section{PROCESSUS DE RIO 1992-1997 \\ CHRONOLOGIE DES FAITS MARQUANTS DE LA CONFÉdÉRATION ET RAPPORTS OFFICIELS}

Juin 1992

jer mars 1993

Printemps 93

Automne 1993

Automne 1994

28 février 1996

18 mars 1996

Juin 1996

Mars 1997

23 au 27 juin 1997

1998

Conférence des Nations Unies sur l'environnement et le développement (CNUED), Rio de Janeiro. UNCED 1992, rapport présenté par la Suisse, OFEFP, avril 1992.

Mise en place du processus IDA-Rio. Le Conseil fédéral confie la coordination du suivi de la CNUED et de la mise en œuvre de l'Agenda 21 à un comité regroupant les directeurs de 17 offices fédéraux, le CIRio (comité interdépartemental). La présidence de ce comité est assumée à tour de rôle par la DDC, I'OFAEE, I'OFEFP, et ce pour une période d'une année. En 1997, le CIRio est présidé par Philippe Roch, directeur de I'OFEFP.

Etablissement d'un groupe de contact et de sept groupes de travail (diversité biologique, forêts, climat, transferts de technologie et coopération technique, incitation et internationalisation des externalités, cohérence de législation et subventions, Forum commerce et environnement). La composition des groupes de travail, réunissant des membres de l'administration fédérale et des secteurs non étatiques (ONG d'environnement et de développement, économie et académie), constitue une nouveauté dans la méthode de travail.

Décision du Parlement de ratifier la Convention sur les changements climatiques.

Décision du Parlement de ratifier la Convention sur la diversité biologique, mais avec une déclaration interprétative réaffirmant l'importance du respect et des règles de propriété intellectuelle.

Le Conseil fédéral prend connaissance du rapport du CIRio Le développement durable en Suisse et donne mandat au CIRio d'élaborer un plan d'action suisse pour le développement durable.

Adoption par le CF de son rapport pour le Plan de législature 1995-1999, qui pour la première fois fait mention de la nécessité pour la Suisse de concrétiser le concept de développement durable.

Le CIRio confie la tâche de rédiger un plan d'action à sept personnalités réunies dans un groupe de travail appelé Conseil pour le développement durable. Son rapport, intitulé Développement durable - Plan d'action pour la Suisse, a servi de base au CIRio pour la rédaction de la stratégie "Le développement durable en Suisse».

Le Conseil fédéral prend connaissance du rapport du CIRio Le développement durable en Suisse - Etat des réalisations et rend publique la Stratégie pour le développement durable en Suisse.

Ces deux documents ont été présentés lors de I'UNGASS.

Session extraordinaire de l'Assemblée générale des Nations Unies sur l'état de la mise en œuvre de l'Agenda 21.

Débat sur le plan d'action suisse et la notion de développement durable au Parlement agendé en 1998.

\section{Collaboration internationale}

La Suisse poursuit ses activités au niveau international dans différentes enceintes:

- elle participe aux réunions de la Commission pour le développement durable qui ont lieu chaque année au printemps à New York;

- elle participe également aux Conférences des parties des conventions biodiversité et climat ainsi qu'aux diverses séances intermédiaires;

- elle dispose d'un siège au Conseil exécutif du GEF et participe financièrement au fonds pour un montant de 64 millions de francs. 


\section{LE DÉVELOPPEMENT DURABLE: DÉFINITION ET INTERPRÉTATION}

¿LE DÉVELOPPEMENT durable satisfait les besoins des générations présentes sans compromettre la possi《bilité pour les générations à venir de satisfaire leurs propres besoins» (définition de la Commission Brundtland).

"La solidarité entre la génération actuelle et les générations futures constitue un aspect central du développement durable. II s'agit de tenir compte d'une part de l'équilibre entre les générations, et d'autre part de celui qui doit exister au sein d'une même génération. Les deux aspects sont importants car ils sont interdépendants. (...)

"Selon la définition donnée par le CIRio, les conditions du développement durable s'appuient sur trois dimensions considérées d'égale valeur: les aspects écologiques, sociaux et économiques.»'

Du point de vue de l'environnement, la notion de développement durable permet de formuler les thèses et les principes suivants:

- Les émissions de polluants doivent être maintenues en deçà de la capacité de la nature à les absorber et à les transformer en substances sans danger.

- La nature doit être préservée dans sa diversité. Les atteintes inévitables doivent être compensées par des mesures qui garantissent l'existence des écosystèmes et la sauvegarde de la diversité biologique.

- Dans un laps de temps donné, les ressources ne doivent pas être épuisées au-delà de leur capacité de régénération.

- Les ressources non renouvelables, par exemple les énergies fossiles, ne doivent pas être épuisées plus rapidement qu'il n'est possible de préparer de nouvelles sources de régénération pour leur développement.

Du point de vue social, la pauvreté de près d'un milliard d'êtres humains, qui ne peuvent couvrir leurs besoins les plus élémentaires, occupe le premier plan. La croissance ininterrompue de la population, les courants migratoires vers les villes, au-delà des frontières nationales et vers d'autres continents, représentent souvent un danger pour la paix sociale. Cette évolution ne peut trouver de réponse sans une amélioration des conditions sociales notamment pour ce qui est du rôle de la femme, ce qui implique aussi des améliorations du logement, des soins médicaux de base, de l'éducation et de l'emploi. Du point de vue social, il est donc urgent de trouver des solutions à ces problèmes.

Du point de vue économique, la croissance est nécessaire pour faire face aux besoins en hausse d'une population mondiale en constante augmentation. De manière analogue à ce que nous avons observé du point de vue environnemental, il faut que notre société vive de ses revenus et non pas de ses réserves en capital. Dans le cadre d'une économie durable, les budgets privés et publics, mais aussi ceux des entreprises doivent s'équilibrer à long terme, de manière à assurer les mêmes chances économiques aux générations futures.

1. Le développement durable en Suisse, rapport du CIRio, février 1996, pp. 9 à 11.

Tiré du Développement durable - Plan d'action pour la Suisse, du Conseil pour le développement durable. 
faire l'inventaire des projets en cours et des décisions récentes qui contribuent déjà à un développement durable en Suisse. Sont notamment mentionnés:

- le programme Energie 2000 et le message relatif à la loi sur les réductions des émissions de $\mathrm{CO}_{2}$ présenté le 17 mars 1997;

- la politique des transports du Conseil fédéral (message du 26 juin 1996), qui tend à rendre le rail plus attrayant que la route et à une meilleure internalisation des coûts externes;

- la réforme de la politique agricole, qui permet d'indemniser mieux la paysannerie pour des prestations écologiques comme la production intégrée et la culture biologique.

ASTM 1996, «Suivi de Rio en Suisse», pp. 53-54.

La stratégie du Conseil fédéral intitulée Le développement durable en Suisse vise à intégrer le développement durable dans toutes les politiques sectorielles de la Confédération. Mais plus spécifiquement, la stratégie a identifié sept domaines qui feront l'objet de mesures complémentaires. Les dispositions ont trait aux huit domaines suivants:

- engagement international, énergie, économie, consommation, politique de sécurité, réforme fiscale écologique, dépenses de la Confédération, mise en œuvre et contrôle.

L'engagement international de la Suisse visera «à un renforcement de ses activités internationales, contribuant activement et solidairement à encourager la durabilité à l'échelle du globe». Et de préciser: «Cet engagement accru profitera également à nos milieux économiques, lesquels ont tout intérêt à ce que l'économie publique des pays en développement prenne un essor durable et à ce que les standards de protection de l'environnement atteignent un niveau plus élevé à l'échelle internationale. $»^{5}$

L'évaluation de la politique de développement durable est reconnue comme indispensable et dans ce but un Conseil pour le développement durable, en tant qu'organe consultatif du Conseil fédéral, sera mis en place courant 1998.

Ces deux documents ont été présentés lors de la session extraordinaire de l'Assemblée générale des Nations Unies sur l'état de la mise en œuvre de l'Agenda 21, qui s'est tenue du 23 au 27 juin 1997 à New York.

\section{Réaction des organisations d'environnement et de développement}

Les principales organisations d'environnement et de développement ${ }^{6}$ saluent les intentions du Conseil fédéral, qui publie la première Stratégie pour le développement durable, et soutiennent la création d'un Conseil pour le développement durable. Mais elles se font très critiques quant aux moyens que le Conseil fédéral se donne pour sa mise en œuvre, notamment à l'égard du manque d'objectifs contrôlables. Les ONG soulignent que «la timidité de la volonté politique du Conseil fédéral se manifeste notamment dans sa proposition de multiplier les études, alors que les données de base sont connues et que les méthodes d'action doivent être définies aujourd'hui sur un plan politique et non pas au niveau des

5. OFEFP, La Suisse sur la voie du développement durable, Berne, 1997, p. 24.

6. Communiqué de presse des organisations d'environnement (SPE, ATE, WWF Suisse et Pro Natura), avril 1997, et conférence de presse de la Communauté de travail du 24 avril 1997, «Cinq ans après Rio, la Suisse est-elle capable de saisir la chance du développement durable?». 
experts». Et de signaler la résignation du Conseil fédéral en matière d'aide publique au développement (l'aide stagne à $0,3 \%$ du PNB alors que la Suisse s'était engagée à la porter à $0,4 \%$ ) ainsi que son peu d'ambition en matière de réforme fiscale (proposition d'étudier différents scénarios d'ici 2001) et au sujet de la réduction des émissions de $\mathrm{CO}_{2}$ d'ici 2010 (le Conseil fédéral garde l'objectif de diminution de $10 \%$ alors que le projet de plan d'action prévoyait une diminution de $20 \%$ ).

De manière générale, les organisations considèrent qu'il y a un manque de volonté politique dans le processus du suivi de Rio et dans la mise en œuvre de l'Agenda 21. Elles rappellent qu'il a fallu attendre quatre ans avant qu'un premier état de la situation sur les enjeux soit publié, et cinq ans pour que le Conseil fédéral propose une véritable Commission pour le développement durable en Suisse. Enfin, elles considèrent que la collaboration entre l'administration et la société civile est encore loin d'être un réel partenariat. Elles relèvent le peu de cas que la Suisse fait de ce thème en signalant le fait que le Parlement a débattu du développement durable - pour la première fois - après la session spéciale de l'Assemblée générale qui marquait le $5^{\mathrm{e}}$ anniversaire du sommet.

\section{$\square$ Commission pour le développement durable (CDD-5)}

La $5^{\text {e }}$ session de la Commission pour le développement durable (CDD-5) ${ }^{7}$ s'est réunie au siège de l'ONU, du 8 au 25 avril 1997, pour parachever les préparatifs de la session extraordinaire de l'Assemblée générale des Nations Unies consacrée à la revue de la mise en œuvre de l'Agenda 21, qui s'est tenue en juin 1997 (voir ci-dessous). L'Egyptien Mostafa Tolba a été nommé à la présidence de la session, Monika Linn Locher, de l'OFEFP, a été nommée vice-présidente pour le groupe Europe de l'Ouest.

La délégation suisse était présidée par Philippe Roch, directeur de l'OFEFP. Des collaborateurs de l'OFEFP, de la DDC, de la Division politique V du DFAE, de l'OFIAMT, de l'OFAEE et de la mission de New York étaient présents. Les organisations d'environnement et de développement étaient représentées par Rosemarie Bär (Communauté de travail des œuvres d'entraide).

La session commença par un segment de haut niveau, pendant lequel des ministres, des ambassadeurs et des ONG présentèrent leurs déclarations. Une série de dialogues avec les principaux groupes sociaux (jeunes, communautés scientifiques, femmes, syndicats, peuples indigènes, etc.) fut entamée parallèlement aux réunions consacrées à la négociation du texte devant être adopté lors de l'Assemblée générale extraordinaire, texte qui dresse les progrès réalisés depuis Rio et détermine les domaines nécessitant une action urgente. D'autre part, le président de la CDD-5, Mostafa Tolba, et la vice-présidente, Monika Linn Locher, entamèrent des consultations sur le projet de déclaration politique à soumettre à la signature des chefs d'Etat et de gouvernement lors du Sommet Rio +5 . Leur approche informelle fut mise en question par un certain nombre de délégations du G77 lors de la plénière de clôture.

Des points importants (déclaration politique, adoption ou pas d'une convention sur les forêts, augmentation des ressources financières) sont finalement restés en

7. Le résumé complet de la CDD-5 peut être consulté sur Internet, Bulletin Négociations de la Terre, http://www. iisd. ca/linkages/ 
suspens. Les discussions ont été reprises lors du Sommet Rio + 5. De manière générale, les négociations ont été plus difficiles que prévu. Certains délégués ont souligné l'incapacité de la CDD à établir des objectifs et des calendriers pour renforcer la mise en œuvre du développement durable, constatant que le résultat ressemble davantage à un rétrécissement de l'interprétation de l'Agenda 21 qu'à sa réaffirmation. Pour les pays en développement, le déclin de l'aide publique au développement depuis 1992 et les tentatives de faire passer la charge du financement international du développement durable à l'investissement du secteur privé, position défendue par les pays industrialisés, ont aidé à discréditer le concept même du développement durable ${ }^{8}$.

\section{$\square$ Sommet Rio +5}

Afin de dresser un premier bilan au plus haut niveau cinq ans après le Sommet planète Terre de Rio, l'ONU a expressément convoqué une session extraordinaire de l'Assemblée générale. La Suisse, non-membre de l'ONU, était tout de même présente avec un statut d'observateur.

SESSION EXTRAORDINAIRE DE L'ASSEMBLÉE GÉNÉRALE DES NATIONS UNIES 1997

Date et lieu

La session extraordinaire a duré une semaine, du 23 au 27 juin 1997, et s'est tenue au siège de l'ONU à New York.

Dresser un bilan intermédiaire de la mise en œuvre du développe-
ment durable et décider de mesures concrètes pour ces prochaines
années.

Bilan

De l'avis général, les résultats de cette session sont extrêmement faibles. Un Programme de mise en œuvre d'une cinquantaine de pages a bien été adopté. Contrairement à ce qui était prévu, la session n'a pas adopté de déclaration politique, mais un texte de consensus intitulé Engagement. De fait, il n'y a pas eu de signal politique clair concernant la protection du climat et des forêts.

Participants

Soixante chefs d'Etat et de gouvernement se sont déplacés pour l'occasion, ainsi que de très nombreux ministres. Les délégations nationales de plus de 164 Etats étaient présentes.

Délégation suisse

La délégation suisse était dirigée par la conseillère fédérale Ruth Dreifuss. Des collaborateurs de I'OFEFP, de la DDC, de la Division politique $V$ du DFAE, de I'OFIAMT, ainsi que la Mission permanente d'observation de la Suisse auprès des Nations Unies étaient également présents. Les organisations d'environnement et de développement étaient représentées par la Communauté de travail des œuvres d'entraide.

Personne de contact pour l'administration

Monika Linn Locher, OFEFP.

8. Brève analyse de la CDD-5, http://www. iisd. ca/linkages/ 


\section{Rencontre ministérielle}

Cent quatre-vingt-sept déclarations sur l'évaluation de la mise en œuvre de l'Agenda 21 ont été faites par des chefs d'Etat, des ministres ou secrétaires d'Etat, mais aussi par des représentants d'organismes internationaux ou d'ONG'. Les orateurs s'accordaient en général pour dire que les principes de l'Agenda 21 sont en train d'être promulgués dans les législations nationales et que deux conventions (biodiversité et climat) signées à Rio sont en cours d'application. Cependant de nombreux intervenants firent remarquer qu'en dépit des engagements pris à Rio, les modes de consommation et de production demeurent à des niveaux incompatibles avec la durabilité. La déforestation se poursuit, aucune volonté politique commune ne se dessine dans des domaines cruciaux tels que les changements climatiques et la gestion de l'eau douce. Enfin, de nombreux pays en développement ne disposent toujours pas des technologies vertes essentielles. Il a été également relevé que l'aide publique au développement continue à décliner, remplacée par les investissements étrangers, fondés sur des objectifs économiques plutôt qu'environnementaux.

Le message de la conseillère fédérale Ruth Dreifuss, cheffe du Département de l'intérieur, se voulait critique, relevant que «beaucoup d'espoirs sont aujourd'hui déçus». Et de citer parmi l'un des échecs les plus cuisants la lutte contre la pauvreté. Ruth Dreifuss a également insisté sur un certain nombre de points défendus par la Suisse. En tant que pays montagneux, la Suisse peut apporter une contribution essentielle dans ce domaine en collaboration avec les pays du Sud ${ }^{10}$. D'autre part elle a insisté sur une meilleure internalisation des coûts sociaux et environnementaux dans les prix. A ce propos la Suisse défend l'idée de l'introduction d'une taxe sur le carburant pour les avions. Enfin, la Conseillère fédérale a rappelé que la Suisse soutient le réapprovisionnement du Fonds pour l'environnement mondial et propose qu'il soit désigné comme mécanisme financier permanent des conventions sur le climat et la biodiversité.

\section{Déclaration d'engagement}

Les 164 Etats ne sont pas parvenus à s'entendre sur une déclaration politique finale. Parmi les questions faisant objet de divergences figuraient la définition du développement durable, les dates butoirs des engagements, les montants attribués à l'aide publique au développement, la mobilisation des ressources intérieures. Les pays en développement lui ont refusé leur soutien, considérant que les pays industrialisés ne favorisaient ni transferts financiers ni transferts technologiques. Les pays industrialisés quant à eux ne souhaitaient en aucune manière s'engager sur un calendrier et des objectifs concrets. N'étant pas parvenue à un accord sur une déclaration politique des chefs d'Etat, la Commission chargée de sa rédaction a, en lieu et place, adopté une «Déclaration d'engagement» de portée politique beaucoup plus faible, dans laquelle un certain nombre de résultats positifs sont admis, tout en reconnaissant que l'état de l'environnement s'est encore dégradé depuis cinq ans.

9. Toutes les déclarations officielles sont disponibles en anglais sur le site officiel du sommet.

10. La Suisse a organisé un séminaire en marge de la session extraordinaire afin de présenter un ouvrage, coproduit par la DDC, l'Université de Berne et la FAO, sur les régions de montagne. 
Programme pour la suite de la mise en auvre de l'Agenda 21

Ce document d'une cinquantaine de pages résume les travaux effectués pendant la $5^{\mathrm{e}}$ Commission pour le développement durable et l'Assemblée extraordinaire de l'ONU. La «Déclaration d'engagement» sert d'introduction, suivie de trois grands chapitres: évaluation des progrès réalisés depuis la CNUED, mise en œuvre dans les domaines nécessitant une action urgente, arrangements institutionnels internationaux. En annexe figure le programme de travail de la Commission pour le développement durable 1998-2002.

Les principaux points du programme:

\section{- Réchauffement climatique}

Aucun consensus ne s'est dégagé pour un plan d'action contre le réchauffement climatique. Les pays européens souhaitaient profiter de la session pour envoyer un message politique fort à la conférence sur le climat qui s'est tenue au Japon en décembre 1997, en réaffirmant leur volonté de réduire les émissions de $\mathrm{CO}_{2}$ de $15 \%$ d'ici 2010. Les Etats-Unis ont refusé de s'engager sur des limitations chiffrées.

- Protection des forêts

Les discussions sur le sujet ont été très difficiles. L'Union européenne, le Canada, le Costa Rica (au nom de l'Amérique centrale) souhaitaient l'ouverture de négociations pour une convention sur les forêts. Les Etats-Unis, le Japon, le G77 et la Chine notamment s'y opposaient. La décision consensuelle finale réside en la création d'un Forum intergouvernemental sur les forêts chargé de mettre en œuvre les décisions du panel intergouvernemental et d'identifier les possibles éléments d'une convention.

- Aide au développement

Les promesses faites à Rio afin d'augmenter l'aide publique au développement n'ont pas été tenues. Les discussions autour de cette question hautement controversée ont abouti à l'idée que l'aide publique reste la principale source de financement pour les PVD, mais qu'elle doit également jouer un effet catalyseur pour la mobilisation de ressources privées.

\section{Position de la Suisse}

De manière générale la position de la Suisse a été présentée par Ruth Dreifuss lors du segment de haut niveau (voir ci-dessus). Plus spécifiquement la Suisse a également insisté sur la dimension sociale du développement durable, notamment en faveur de la création d'emplois, rappelant les engagements pris lors du Sommet mondial pour le développement social de Copenhague (1995). A noter cependant que si la Suisse, non-membre des Nations Unies, a pu participer à titre d'observateur aux débats, elle n'a pas eu le droit de vote. Une Suissesse, Monika Linn Locher, cheffe de section des affaires internationales de l'OFEFP, avait pourtant été nommée vice-présidente de la $5^{\mathrm{e}}$ Commission pour le développement durable.

\section{Evaluation du Sommet Rio +5}

Pour les ONG et les médias, les résultats du Sommet Rio + 5 sont faibles, mais étaient prévisibles après les difficiles négociations qui avaient eu lieu lors de la préparation de la Conférence (CDD-5). L'échec politique de cette session extraordinaire de l'ONU est important, car la démonstration d'une volonté ferme des 
gouvernements dans la mise en œuvre de l'Agenda 21 était indispensable pour coordonner les initiatives et stimuler les énergies de la société civile.

\title{
RAPPORT DU PROGRAMME DES NATIONS UNIES POUR L'ENVIRONNEMENT "APERCUU SUR L'ENVIRONNEMENT MONDIAL"
}

\begin{abstract}
L'OCCASION du Sommet Rio + 5, le PNUE a rédigé un rapport dressant un état des lieux de la situation Aenvironnementale au niveau de la planète.

Quelques progrès ont été réalisés en termes de développement institutionnel, de renforcement du consensus international, de participation publique et d'actions à l'initiative du secteur public; ainsi quelques pays ont réussi à ralentir le rythme de la dégradation des ressources. Les taux de croissance démographique ont baissé d'une manière générale, en grande partie grâce à l'expansion de l'éducation de base et des soins de santé primaires. Mais en dépit de ces acquis, les tendances de la situation générale sont en train d'empirer. L'augmentation des niveaux de pollution menace d'excéder la capacité d'absorption de l'environnement mondial.
\end{abstract}

Une version anglaise du rapport "Global Environment Outlook" peut être consultée sur Internet: http://www. grid. unep. ch/geo1/index. htm

\section{$\square$ Concrétisation de la notion de développement durable}

«Satisfaire les besoins des générations actuelles sans hypothéquer les chances des générations à venir» est la formule de base autour de laquelle doit se concrétiser le développement durable. En Suisse, l'OFEFP a lancé un vaste programme pluriannuel de soutien à des projets de mise en œuvre du plan d'action, et les organisations d'environnement et de développement ont fourni, en 1997, quelques contributions importantes à la concrétisation de la notion de développement durable.

\section{Campagne Nord/Sud pour le développement durable}

La Communauté de travail Swissaid/Action de Carême/Pain pour le prochain/Helvetas/Caritas a lancé le 30 janvier 1997 une vaste opération intitulée «Campagne Nord/Sud pour le développement durable», qui durera jusqu'en 1998 et qui sera ponctuée par différents événements. Le document de référence de la campagne est le Manifeste Nord/Sud. Il contient 21 thèses pour le développement durable qui définissent le rôle que la Suisse devrait jouer au niveau international et les possibilités d'action des différents acteurs: milieux politique et économique, société civile et consommateurs"

Dans le cadre de sa campagne, la Communauté de travail a conduit une vaste enquête ${ }^{12}$ auprès d'un échantillon représentatif en Suisse, qui a révélé qu'un tiers (34\%) de la population pouvait expliquer correctement la notion de développement durable; $25 \%$ par contre en étaient incapables. Plus de la moitié des personnes interrogées considère que le développement durable concerne aussi bien le Nord que le Sud et que les efforts déployés dans ce sens par les pouvoirs publics, l'économie et les citoyens suisses sont encore insuffisants.

11. Une publication de la Communauté de travail, Revue Sud, "Que signifie le développement durable?», dresse un tableau de la situation au niveau mondial, expose les objectifs de la campagne et présente le Manifeste Nord/Sud. Disponible au secrétariat de la CT, tél. 021/617.43.53.

12. Enquête Isopublic, conduite à la demande de la Communauté de travail sur le développement durable, janvier 1997. 
«Environnement, économie, société:

18 thèses au sujet du développement durable»

La contribution des organisations d'environnement s'est faite par la publication d'une brochure bilingue, Environnement, Economie, Société: 18 thèses au sujet $d u$ développement durable ${ }^{13}$. Initialement l'ATE, la SPE, le WWF et Pro Natura avaient élaboré un certain nombre de propositions conformes au développement durable en vue des débats relatifs à la réforme de la Constitution fédérale. L'objectif initial n'a pas été abandonné, mais il a été complété. Ainsi la brochure contient une série de propositions concernant:

- l'Etat: son rôle et l'importance d'intégrer la notion de durabilité dans la gestion du pays;

- les aspects économiques et sociaux: une importance particulière est portée au travail, au revenu et à la fiscalité;

- l'environnement et la technique: sont prônés une utilisation rationnelle des ressources naturelles, la protection de l'environnement et du paysage, un aménagement du territoire rationnel, une réduction des transports pour les individus et pour les marchandises;

- la mise en œuvre et le contrôle: les normes et l'action de l'Etat doivent être harmonisées et un système de plaintes à l'encontre des pouvoirs exécutifs et législatifs en cas de non-respect d'un mandat inscrit dans la Constitution doit être introduit.

C'est au niveau des propositions d'ordre juridique que cette publication est le plus originale; pour le reste elle reprend les thèmes traditionnels du développement durable.

\section{«Das Existenz-Maximum - Grundlagen für eine zukunftsfähige Schweiz»}

S'appuyant sur les gestes de la vie quotidienne, le livre ${ }^{14}$ de la Déclaration de Berne souhaite motiver chaque lecteur afin qu'il opte pour des choix de consommation qui soient compatibles avec la notion de durabilité. L'ouvrage présente également différents aspects des relations Nord/Sud, qui ont des impacts dans les domaines environnementaux, sociaux et économiques.

\section{"Comprendre la norme ISO 14'001»}

Les entreprises ont un grand rôle à jouer dans la concrétisation du développement durable. Dans le prolongement du Sommet de Rio, les milieux économiques ont proposé la mise sur pied d'une norme internationale ISO 14'001, qui a été approuvée en 1996. La norme ISO 14'001 établit les lignes directrices de la mise en place d'un système de management environnemental. Celui-ci permet à une entreprise de déterminer tous ses impacts sur l'environnement, d'identifier les améliorations possibles et de les mettre en œuvre. La SPE a édité une brochure ${ }^{15}$, guide d'initiation à la norme ISO 14'001, qui permet de comprendre les exigences de la mise en place d'un tel système. Au niveau mondial, le nombre de

13. Pro Natura, SPE, WWF Suisse, ATE, Environnement, économie, société: 18 thèses au sujet du développement durable, une contribution des organisations d'environnement, mai 1997.

14. Déclaration de Berne, Das Existenz-Maximum - Grundlagen für eine zukunftsfähige Schweiz, Zurich, 1997. Disponible uniquement en allemand auprès du secrétariat de la Déclaration de Berne, tél. 01/271.64.34.

15. Société pour la protection de l'environnement, Comprendre la norme ISO 14'001, SPE, septembre 1996. Disponible uniquement en français auprès du secrétariat de la SPE, tél. 022/329.99.29. 
certificats selon la norme ISO est en augmentation rapide et en Suisse on compte déjà plus de 100 firmes certifiées (des industries lourdes aux fabricants de cosmétiques, en passant par les établissements bancaires). La validité du certificat n'est que de trois ans; passé ce délai un nouvel examen doit être entrepris.

\section{SOURCES}

\section{Suivi de Rio en Suisse et Rio + 5}

Conseil fédéral, Le développement durable en Suisse - Stratégie, Berne, avril 1997.

CIRio, Le développement durable en Suisse - Etat des réalisations, rapport mis à jour en avril 1997, Berne, avril 1997.

Conseil pour le développement durable, Développement durable - Plan d'action pour la Suisse, OFEFP, mars 1997.

OFEFP, La Suisse sur la voie du développement durable, Berne, 1997.

OFEFP, Communiqué de presse, 14 avril 1997, «La Suisse doit persévérer en matière de développement durable».

OFEFP, Environnement, bulletin de l'OFEFP, «Développement durable - Cinq ans après le Sommet de la Terre à Rio», $2 / 97$.

OFEFP, Environnement, bulletin de l'OFEFP, «Bilan suisse cinq ans après Rio: une lente prise de conscience», $3 / 97$.

Earth Negotiations Bulletin, vol. V, $\mathrm{n}^{\circ} 82$ (site http://www. iisd. ca/linkages/)

Communauté de travail, Dossier de presse, 24 avril 1997, «Cinq ans après Rio, la Suisse est-elle capable de saisir la chance du développement durable?».

Communauté de travail, Swiss Coalition News, «Rio + 5, More than Sustainable Illusions?», $\mathrm{n}^{\circ}$ 12, May 1997.

SPE, ATE, WWF Suisse et Pro Natura, Communiqué de presse des organisations d'environnement, avril 1997, « Plan d'action du Conseil fédéral pour le développement durable - Trop peu, trop tard et si timide... dommage ».

Journal de Genève, 5 juin 1997 «Philippe Roch: j'ai appris à comprendre les exigences de l'économie», 23 juin 1997 «Les Terriens se retrouvent à New York», 28 juin 1997 «Au Sommet de la Terre, l'esprit de Rio est retombé comme un soufflém.

La Liberté, 25 juin 1997 «Pour sauver la Terre, il faut dépasser les divergences politiques», 26 juin 1997 «Quelle organisation pourrait sauvegarder l'environnement?», 27 juin 1997 «Le Sommet de la Terre menace de capoter sur la question des gros sous".

Le Monde, 24 juin 1997 «L'environnement, source de discorde au Sommet du G8», 28 juin 1997 «L'environnement s'est dégradé depuis cinq ans".

Neue Zürcher Zeitung, 5. Juni 1997 «Rio-Zwischenbilanz nach fünf Jahren», 24. Juni 1997 «Erdgipfel plus fünf in New York eröffnet», 25, Juni 1997 «Ruth Dreifuss am New Yorker Erdgipfel», 28. Juni 1997 «Selbskritik der USA am Erdgipfel in New York» und «Fünf Jahre nach Rio - Die Bilanz bleibt offen».

Tages Anzeiger, 28. Juli 1997 «Im Gespräch, Monika Linn Locher zum Umweltgipfel».

Concrétisation de la notion de développement durable

Communauté de travail, Revue Sud, «Que signifie le développement durable?», 2/1997.

Communauté de travail, Swiss Coalition News, «North-South Manifesto for Sustainable Development», n¹1, February 1997.

Pro Natura, SPE, WWF Suisse, ATE, Environnement, Economie, Société: 18 thèses au sujet du développement durable, une contribution des organisations d'environnement, mai 1997.

Neue Zürcher Zeitung, 4 März 1997 « Mehr Okologie in den öffentlichen Verwaltungen ».

Déclaration de Berne, Das Existenz-Maximum - Grundlagen für eine zukunftsfähige Schweiz, Zurich, 1997.

Société pour la protection de l'environnement, Comprendre la norme ISO 14001, SPE, septembre 1996.

\section{SITE INTERNET}

Site officiel de la session extraordinaire de l'Assemblée générale de l’ONU (Rio + 5): http://www. un. org/DPCSD/earthsummit/ 


\subsection{PRÉSERVATION ET CONSERVATION DURABLE DE LA DIVERSITÉ BIOLOGIQUE}

Les activités dues à l'intervention humaine menacent de plus en plus la diversité biologique (les espèces animales et végétales, leur matériel génétique et les écosystèmes dont elles font partie). L'accumulation des pollutions, les pratiques agricoles non durables, les déforestations massives, les changements climatiques sont parmi les facteurs qui contribuent à l'érosion de la biodiversité en perturbant l'écosystème et en détruisant les habitats.

\section{LES CONVENTIONS INTERNATIONALES POUR LA PROTECTION DE LA BIODIVERSITÉ}

- Convention internationale du 2 décembre 1946 pour la réglementation de la chasse à la baleine

- Convention du 18 octobre 1950 sur la protection des oiseaux

- Convention du 2 février 1971 relative aux zones humides d'importance internationale, particulièrement comme habitat des oiseaux d'eau (convention Ramsar)

- Convention du 3 mars 1973 sur le commerce international des espèces de faune et de flore sauvages menacées d'extinction (CITES)

- Convention du 23 juin 1979 sur la conservation des espèces migratrices appartenant à la faune sauvage (convention de Bonn)

- Convention du 19 septembre 1979 relative à la conservation de la vie sauvage et du milieu naturel d'Europe (convention de Berne)

- Convention du 5 juin 1992 sur la diversité biologique (Convention de Rio)

\section{$\square$ La $3^{e}$ Conférence des parties de la Convention sur la biodiversité (COP-3)}

Précédée par un certain nombre d'ententes légales sur la protection des espèces et de l'habitat, la Convention sur la diversité biologique de 1992 constitue le plus récent instrument multilatéral conçu pour répondre à cette crise écologique. La convention, entrée en vigueur en décembre 1993, a pour objectifs la conservation de la diversité biologique, l'utilisation durable des ressources biologiques et une répartition juste et équitable des bénéfices de l'utilisation des ressources génétiques. Depuis l'entrée en vigueur de la Convention sur la biodiversité, les Etats parties à la convention se sont réunis trois fois lors de conférences internationales, appelées Conférences des parties (COP). La COP-1 a établi un programme de travail à moyen terme et décidé de la création d'un groupe de travail spécifique: un organe subsidiaire chargé de fournir des avis scientifiques, techniques et technologiques (plus connu sous l'abréviation SBSTTA). Lors de la COP-2, il a été décidé du choix du secrétariat de la convention à Montréal, de la rédaction d'un protocole sur la biosécurité devant compléter la convention et de considérer le Fonds pour l'environnement mondial (FEM/GEF) comme mécanisme de financement intérimaire. La situation de la biodiversité côtière et marine a été abordée, ainsi que la question des forêts comme réservoir de biodiversité.

La $3^{\text {e }}$ Conférence des parties (COP-3) a précisé son fonctionnement, ainsi que son rôle et ses liens avec d'autres instruments internationaux, notamment ceux relatifs aux droits de propriété intellectuelle, aux forêts et à l'Agenda 21. Dans ce processus, la Conférence des parties a commencé à se frayer son chemin en essayant d'affirmer son autorité sur le Fonds pour l'environnement mondial 
(FEM) et d'asseoir son autonomie par rapport au PNUE, en affinant son programme de travail et son plan d'action futur. Durant la conférence, les délégués ont pris plusieurs mesures importantes: l'élaboration d'un programme de travail en matière de diversité agricole et d'un programme, plus limité, dans le domaine de la biodiversité forestière; un mémorandum d'accord avec le FEM longuement négocié; l'organisation d'un atelier sur le savoir, les innovations et les pratiques des communautés indigènes et locales durant la période intérimaire; la demande d'un statut d'observateur à la Commission de l'OMC chargée du commerce et de l'environnement; enfin elle a préparé une déclaration à l'intention de la session extraordinaire de l'Assemblée générale de l'ONU consacrée à la revue de la mise en œuvre de l'Agenda 21.

TROISIÈME CONFÉRENCE DES PARTIES À LA CONVENTION SUR LA DIVERSITÉ BIOLOGIQUE - COP-3

Date et lieu

La conférence s'est tenue du 4 au 15 novembre 1996, à Buenos Aires.

Thèmes principaux

Discussions sur les recommandations de la deuxième réunion de la SBSTTA; évaluation du mécanisme d'échange d'informations; ressources financières et mécanisme de financement (FEM/GEF); mesures générales pour la conservation et l'utilisation durable; biodiversité agricole; programme de travail en matière de biodiversité terrestre; savoir, innovations et pratiques des communautés indigènes et locales; accès aux ressources génétiques; questions liées aux transferts technologiques; droit de propriété intellectuelle; biosécurité.

Participants

Les délégations des 160 pays ayant ratifié la convention ont participé aux travaux de la COP-3. Une réunion ministérielle s'est tenue les 13 et 14 novembre 1996, pendant laquelle les délégués ont pu entendre plus de 80 déclarations de gouvernements, d'organisations internationales et d'ONG. Prirent également la parole: la présidente de la COP-3 Maria Julia Alsogaray, la directrice exécutive du PNUE Elizabeth Dowdeswell et le président argentin Carlos Menem.

Délégation suisse

La délégation suisse de la COP-3 était dirigée par Philippe Roch, directeur de I'OFEFP. La délégation était composée de Louis Curat (DDC), qui s'est vu confier la vice-présidence de la conférence, Robert Lamb et François Pythoud (OFEFP), Jürg Benz (DDC), Benno Bättig (OFAEE) et Lorenza Ferrari (IFPI).

Philippe Roch, OFEFP.

Ressources financières et mécanisme de financement

Un document, faisant appel à toutes les institutions de financement multilatérales et bilatérales afin qu'elles répondent mieux aux besoins de la convention, a été adopté sans difficulté majeure.

Par contre un mémorandum d'accord entre la Conférence des parties et le Fonds pour l'environnement mondial fut âprement négocié et note que le FEM fonctionnera encore sur une base intérimaire jusqu'à la prochaine Conférence des parties. Les points de discussion concernent: 
- les possibilités pour la Conférence des parties d'agir comme instance de recours contre les décisions du FEM;

- l'évaluation des projets «biodiversité» à financer par le Fonds;

- la discussion sur l'évaluation de l'efficacité du FEM qui se fera lors de la COP-4;

- les domaines nouveaux que la conférence souhaiterait voir financer par le Fonds (biodiversité agricole, taxonomie, sécurité biologique, mécanisme d'échange pour les questions de propriété intellectuelle et de participation des populations indigènes, etc.).

Les débats sur ces différents points ont montré qu'il existe encore de fortes tensions entre le FEM et la Conférence des parties.

La Suisse, conjointement avec l'Argentine, a organisé deux séminaires sur le FEM pendant la Conférence des parties, ainsi qu'un voyage en Patagonie qui a permis à 60 participants de visiter un projet financé par le FEM. Cette visite a donné lieu à une meilleure connaissance du fonctionnement du FEM et à l'ouverture d'un dialogue entre les différents partenaires: le Directeur exécutif du FEM, ses collaborateurs et les représentants des délégations officielles et des ONG.

\section{La biodiversité agricole}

La biodiversité dans l'agriculture était l'un des points prioritaires de la session. Dans ce domaine, il s'agissait de renforcer les synergies avec les travaux d'autres forums internationaux, en particulier ceux menés au sein de la FAO, et de favoriser la prise en compte des objectifs de la convention dans les politiques agricoles.

\section{Savoir, innovations et pratiques des communautés indigènes et locales}

La discussion sur cette question a été enrichie par la présence de représentants des peuples indigènes de l'Amérique latine, de la région pacifique et de l'Afrique, appuyés par plusieurs pays en développement. Dans son intervention, la Suisse a insisté sur le rôle important des populations autochtones et sur leurs savoirs traditionnels pour la conservation et l'utilisation durable de la diversité biologique, ainsi que sur la nécessité d'établir un régime qui permette de compenser les innovations traditionnelles des populations indigènes et des communautés locales. Un atelier de travail sur cette question a été organisé en novembre 1997 afin de préparer une documentation générale sur ce thème qui fera l'objet d'une discussion approfondie lors d'une prochaine Conférence des parties.

\section{Droits de propriété intellectuelle}

La discussion sur les droits de propriété intellectuelle reflète l'insatisfaction qui règne parmi certaines délégations et certaines $\mathrm{ONG}$ quant au traitement accordé à cette question dans les accords TRIPS de l'OMC. Parmi les sujets de préoccupation: l'absence d'un régime de propriété intellectuelle reconnaissant le savoir traditionnel, certaines formes d'octroi de brevets pour des produits dérivés de la biotechnologie et le rôle de l'OMC en matière de régulation mondiale de la propriété intellectuelle. A ce propos, la Conférence des parties a décidé de demander le statut d'observateur auprès du Comité sur le commerce et l'environnement de l'OMC. 


\section{Rédaction d'un protocole sur la biosécurité}

Les potentialités offertes par les biotechnologies modernes dans les secteurs tels ceux de la santé, l'agriculture ou l'environnement ne pourront être concrétisées que dans un cadre global garantissant un niveau élevé de sécurité pour l'homme et l'environnement. Ainsi, en 1995, des négociations ont été lancées pour élaborer un protocole sur la prévention des risques liés aux biotechnologies. La Conférence des parties a pris connaissance de l'avancement des travaux du groupe de travail ad hoc sur la biosécurité, chargé d'élaborer ce protocole qui compléterait la convention sur ce point. Les discussions concernant le projet du protocole devraient commencer en 1998.

\section{Position suisse à la COP-3}

Parmi les thèmes centraux de la conférence, la Suisse s'est particulièrement engagée pour l'amélioration des relations entre la Conférence des parties et le mécanisme financier de la convention qui est le Fonds pour l'environnement mondial (FEM). Elle a également participé activement aux discussions sur les forêts, la sécurité en matière de biotechnologie, les connaissances traditionnelles des populations indigènes, ainsi que sur les questions relatives à l'accès aux ressources génétiques et au partage des bénéfices de l'utilisation de ces ressources. Elle s'est notamment exprimée pour renforcer la participation du secteur privé dans le développement des travaux sur les mesures économiques qui permettent de contribuer à la conservation et à l'utilisation durable de ces ressources.

Dans la Déclaration de la Suisse à la partie ministérielle de la COP-3, Philippe Roch a mentionné certains des points soutenus par la Suisse et a insisté vigoureusement sur la nécessité d'améliorer les conditions de travail de la Conférence des parties (meilleure définition des objectifs, limiter le nombre de documents et de groupes plus ou moins formels, prolonger les délais de travail pour fournir de meilleures analyses) $)^{16}$.

La 4 Conférence des parties se tiendra à Bratislava, en Slovaquie, du 4 au 15 mai 1998. Les dispositions de la Convention sur le partage équitable des ressources seront un des points centraux de la conférence. Ce sujet est complexe et politiquement délicat: d'une part ces dispositions ne sont pas encore assez concrétisées, d'autre part leur interprétation fait l'objet de divergences entre les pays du Nord et ceux du Sud (accès aux ressources génétiques, accès aux biotechniques, valorisation du savoir des peuples autochtones et des communautés paysannes).

ASTM 1995, pp. 62-64 (ratification de la convention par la Suisse avec une déclaration interprétative de la Suisse). ASTM 1997, pp. 55-59 (présentation du texte de la convention, COP-1, COP-2).

16. Déclaration de la Suisse, faite par Philippe Roch, directeur de l'OFEFP, à la partie ministérielle de la $3^{\circ}$ Conférence des parties à la Convention sur la diversité biologique (Buenos Aires, le 13 novembre 1997). 


\section{SOURCES}

Convention des Nations Unies sur la diversité biologique.

Message concernant la Convention des Nations Unies sur la diversité biologique.

United Nations Environment Programme, Rapport de la 3e réunion de la Conférence des parties à la Convention sur la diversité biologique, UNEP/CBD/COP/3/38, 11 février 1997.

OFEFP, Environnement, bulletin de l'OFEFP, «Développement durable - Cinq ans après le Sommet de la Terre à Rio, biodiversité», pp. 27 à 34, 2/97.

OFEFP, Communiqué de presse, «Conférence des parties à la Convention sur la diversité biologique, participation active de la Suisse», 15 novembre 1996.

Earth Negotiations Bulletin, $3^{\text {e }}$ session de la Conférence des parties à la Convention sur la diversité biologique, 4-15 novembre 1997, vol. 09: 65, disponible sur Internet: http://www. iisd. ca/linkages/

Ecodécision, «Biodiversité, sociodiversité et aires protégées», n²3, hiver 1997.

Journal de Genève, 5 novembre 1997 «La Suisse défend la biodiversité en Argentine».

Neue Zürcher Zeitung, 15. Januar 1997 «Wann ist der Schutz der Biodiversität nachhaltig?».

\section{SITES INTERNET}

Official Web site of the Convention on biological Diversity: http://www. biodiv. org/

Earth Negotiations Bulletin (electronic clearing-house for information on past and upcoming international meetings related to environment and development: http://www. iisd. ca/linkages. Version française: http://www. iisd. ca/linkages/french

\section{- Convention sur le commerce international} des espèces de faune et de flore sauvages menacées d'extinction (CITES)

La CITES $^{17}$ (Convention sur le commerce international des espèces de faune et de flore sauvages menacées d'extinction) est une convention commerciale internationale, dont l'objet n'est pas d'interdire le commerce d'animaux ou de plantes sauvages, mais de l'organiser de telle façon que la survie de ces espèces ne soit pas menacée. La Suisse joue un rôle important en tant qu'Etat dépositaire de la convention et en tant que pays siège du secrétariat à Genève ${ }^{18}$. En vigueur depuis juillet 1975, la convention est applicable dans 140 Etats. Harare, capitale du Zimbabwe, a accueilli du 9 au 20 juin 1997 la $10^{\circ}$ Conférence des parties à la CITES. La délégation suisse a été conduite par Peter Dollinger, chef de la division du trafic international à l'Office vétérinaire fédéral (OVF).

A l'ordre du jour de la session figuraient de nombreuses propositions d'amendements des annexes de la convention. En effet, les dispositions de la convention sont applicables à quelque 4000 espèces animales et à plus de 30'000 espèces de plantes. Les espèces sont réparties dans trois annexes selon la réglementation qui leur est applicable:

- Annexe I: espèces menacées d'extinction qui pourraient être affectées par le commerce international;

- Annexe II: espèces qui pourraient être menacées d'extinction si leur commerce n'était pas sévèrement réglementé et contrôlé;

- Annexe III: espèces pour lesquelles des règles spéciales sont applicables lorsqu'elles ont été désignées par une partie.

Ce sont ainsi plus de 70 espèces, dont la situation a été évaluée et qui ont changé, inclus ou biffé de positionnement dans les annexes.

Dans l'évaluation des espèces deux points de vue s'affrontent entre les partisans de l'exploitation durable et ceux de la protection absolue. Les premiers estiment

17. Convention on International Trade in Endangered Species of Wild Fauna and Flora.

18. Secrétariat CITES, chemin des Anémones 15, case postale 456, 1219 Châtelaine - Genève. Tél. 022/979.91.39, fax 022/797.34.17. 
que le plus sûr moyen d'assurer l'avenir des espèces animales est de leur donner une valeur financière, ce qui suppose la commercialisation. Les seconds soulignent que les règles de l'économie ne peuvent conduire qu'à une exploitation abusive et à l'extinction des espèces. La position de la Suisse s'établit sur une évaluation du double principe de la conservation et de l'exploitation durable, en tenant compte des critères préétablis par la CITES.

Parmi les quelque 70 espèces évaluées, trois ont retenu l'attention de l'opinion publique: les éléphants d'Afrique, les baleines et les rhinocéros blancs. Après négociations pour ces deux derniers le statut n'a pas changé. Ce n'est pas le cas en revanche de l'ivoire, dont l'embargo installé depuis 1990 a été levé à la demande de trois pays (Zimbabwe, Botswana et Namibie). Ceux-ci ont obtenu l'autorisation de vendre une partie de leur stock d'ivoire au Japon après un moratoire de dix-huit mois. L'abstention de l'Union européenne a permis la levée de l'embargo, la Suisse ayant voté en faveur d'un commerce contrôlé de l'ivoire.

Greenpeace, qui reproche à la Suisse une interprétation de la convention qui favorise plus la protection du libre commerce que celle des espèces, a lancé une virulente campagne demandant la démission du représentant suisse, Peter Dollinger. Une pétition, munie de 15'000 signatures, a été présentée au Conseil fédéral en août 1997. Le WWF Suisse regrette la décision prise à Harare de lever l'embargo sur le commerce d'ivoire, la trouvant prématurée; cependant l'organisation s'est nettement distancée de la campagne de Greenpeace.

Convention sur le commerce international des espèces de faune et de flore sauvages menacées d'extinction (CITES). Office vétérinaire fédéral, La Suisse et la CITES, OVF, juin 1997.

Département fédéral de l'économie publique, Communiqué de presse, "Conférence sur la conservation des espèces», 2 juin 1997.

CITES, Press Note, août 1997.

Greenpeace, Communiqué de presse, «Virez Dollinger - Protégez les espèces», Berne, 14 juillet 1997.

La Liberté, 17 juin 1997 «Une semaine décisive pour l'éléphant», 21 juin 1997 «L'ivoire pourra à nouveau être l'objet de commerce».

Le Monde diplomatique, «Quelle rentabilité pour la faune sauvage?», juin 1997.

Neue Zürcher Zeitung, 10. Juni 1997 «Streit um Elfenbeinhandel im Südlichen Afrika», 15. Juli 1997 «Greenpeace auf Kopfjagd». 


\subsection{MISE EN CEUVRE DE LA CONVENTION SUR LES CHANGEMENTS CLIMATIQUES}

L'enjeu de la conférence sur le climat était sans précédent. Le rapport scientifique du Groupe d'experts intergouvernemental sur l'évolution du climat présenté en décembre $1995^{19}$ ne laisse guère de doute: le réchauffement est une réalité établie, ses causes résident en partie dans la combustion des énergies fossiles (pétrole et charbon) et dans les pratiques agricoles et forestières intensives. La seule incertitude réside dans l'amplitude des conséquences. La communauté internationale s'est réunie pour la $3^{e}$ Conférence des parties à la Convention sur le climat pour tenter d'inverser la tendance à la hausse des rejets de gaz à effet de serre. La conférence de Kyoto se présentait comme une difficile phase de négociations et ce fut bien le cas. C'est in extremis que les Etats sont parvenus à un accord, le "protocole de Kyoto».

L'objectif ultime de la convention défini dans son article 2 est «de stabiliser les concentrations de gaz à effet de serre dans l'atmosphère à un niveau qui empêche toute perturbation anthropique dangereuse du système climatique». La COP-1, qui s'était tenue à Berlin, a jugé insuffisant l'engagement des pays industrialisés pour atteindre cet objectif. Elle a donc décidé, en adoptant le Mandat de Berlin, de prescrire des objectifs quantifiés de réduction des émissions de gaz à effet de serre pour les pays industrialisés selon des échéances précises. Le groupe de travail ad hoc du Mandat de Berlin a donc été chargé d'élaborer un protocole (ou un autre instrument juridique) pour préciser l'engagement des pays industrialisés.

Les différents Etats ou groupes d'Etats parties à la négociation sont arrivés avec des positions divergentes et c'est par un véritable tour de force que la conférence ne s'est pas conclue sur un échec total. L'Union européenne exigeait que les pays industrialisés réduisent leurs émissions de gaz à effet de serre de 7,5\% d'ici à 2005 et de $15 \%$ d'ici à 2010 par rapport à leur niveau de 1990. Elle proposait que les pays industrialisés adoptent des politiques et des mesures contraignantes et harmonisées pour atteindre ces objectifs. Les Etats-Unis, le plus grand émetteur mondial de gaz à effet de serre, sont arrivés au Japon en faisant connaître leur intention de stabiliser leurs émissions d'ici à 2008-2012 et de ne les réduire qu'après ces dates. Opposés aux mesures contraignantes et harmonisées avec d'autres pays industrialisés, ils préconisaient l'introduction d'instruments économiques. Le Japon et les pays d'Europe centrale et orientale avaient une position plus ambitieuse que les Etats-Unis en matière de réduction puisqu'ils avaient annoncé leur intention de réduire leurs émissions de 5\% d'ici à l'an 2008. Le traditionnel clivage Nord-Sud a refait surface à Kyoto puisque les pays en développement et la Chine ont rappelé que le problème de l'effet de serre a été créé par les pays industrialisés et que c'est à eux de le résoudre. Il est vrai que le Mandat de Berlin n'impose pas aux pays du Sud de nouvelles obligations. Si les pays en développement émettent actuellement moins de gaz à effet de serre que les pays industrialisés, le volume de leurs émissions devrait dépasser celui des pays industrialisés en l'an 2010.

19. GIEC, Deuxième rapport d'évaluation du GIEC - Changements climatiques, 1995, Genève, OMM-PNUE, décembre 1995. 
TROISIĖME CONFÉRENCE DES PARTIES À LA CONVENTION SUR LES CHANGEMENTS CLIMATIQUES COP-3

Date et lieu La conférence s'est tenue du $1^{\text {er }}$ au 10 décembre 1997, à Kyoto.

Objectifs principaux

L'objectif central de la conférence consistait en la négociation d'un protocole prescrivant, pour les pays industrialisés, des objectifs contraignants de réduction de gaz à effet de serre selon des échéances précises $(2005,2010,2020)$.

D'autres points importants figuraient également à l'ordre du jour:

$\checkmark$ adoption du règlement interne de la COP;

\ amendement des articles 4.3 (élargissement des compétences financières) et 17 (fixation du système majoritaire pour l'approbation du protocole);

$\checkmark$ réévaluation de la liste des pays à qui incombent des obligations spéciales (annexes I et II);

$\checkmark$ adoption du budget de la convention;

$\checkmark$ discussion du mécanisme financier de la convention afin de soutenir les pays en développement dans leurs efforts de réduction de leurs émissions de gaz à effet de serre.

Participants

La plupart des 167 Etats parties à la convention étaient représentés lors du sommet de Kyoto. Un segment ministériel s'est tenu du 8 au 10 décembre, pendant lequel les chefs de gouvernement et ministres ont conclu les négociations.

Délégation suisse

La délégation suisse à la COP-3, dirigée par Beat Nobs, de I'OFEFP, était composée de collaborateurs des offices fédéraux de l'environnement, des forêts et du paysage, de l'énergie, des affaires étrangères, des affaires économiques extérieures. Des milieux extérieurs à l'administration ont été invités (Vorort, WWF Suisse et représentants des milieux académiques). La conseillère fédérale Ruth Dreifuss et le directeur de l'OFEFP, Philippe Roch, représentaient la Suisse lors du segment ministériel.

Contact pour l'administration

José Romero, Division des affaires internationales, OFEFP

tél. 031/322.68.62.

Markus Nauser, Unité Changement climatique, OFEFP

tél. 031/324.42.80.

\section{Les résultats de la conférence de Kyoto}

Les dix jours de négociations ont été extrêmement difficiles et c'est in extremis que les quelque 160 pays sont parvenus à un accord qui prend la forme d'un protocole juridiquement contraignant. Pour la première fois des objectifs de réduction chiffrés ainsi qu'un calendrier ont été acceptés par les Etats. Mais le protocole contient également des références à des instruments économiques (permis négociables, Joint Implementation et Clean Development Mechanism), qui ont été introduits sous la très forte pression des Etats-Unis.

Les pays industrialisés ont donc accepté de réduire leurs émissions de 5,2\% en moyenne d'ici à 2008-2012 par rapport à leur niveau de 1990. Cette réduction totale sera obtenue par une réduction différenciée selon les pays: $8 \%$ pour la 
Suisse, l'Union européenne et la plupart des pays d'Europe centrale et orientale; 7\% pour les Etats-Unis; $6 \%$ pour le Canada, la Hongrie et le Japon. Par contre, certains pays se voient autorisés à augmenter leurs émissions (1'Australie de 8\% et la Norvège de $1 \%$ ).

Le protocole autorise de nouveaux instruments économiques. Les «permis négociables», plus communément connus sous le terme "permis de polluer», permettront aux pays qui dépasseront leur plafond de racheter des droits à ceux qui n'auraient pas épuisé la totalité de leur quota d'émissions. La Joint Implementation (mise en œuvre conjointe) est une forme de collaboration entre les pays qui permet à chacun d'entre eux d'opérer une partie de sa réduction par des investissements privés ou publics engagés dans des projets réalisés dans un autre pays. Cet instrument devrait améliorer le rapport coûts-bénéfices des investissements ayant des répercussions sur le climat; il devrait également favoriser un engagement accru du secteur privé. Le Clean Development Mechanism est le mécanisme qui permettra la mise en œuvre conjointe entre les pays industrialisés et les pays en développement. Si le principe de la création de ces instruments a été accepté à Kyoto, les mécanismes de fonctionnement de ce nouveau marché n'ont été ni définis ni adoptés. Ils le seront lors de la prochaine conférence sur le climat, prévue à Buenos Aires en novembre 1998. D'ici là, aucun pays ne pourra négocier de tels droits.

Dans sa forme finale, le protocole satisfait d'une part l'Union européenne, qui souhaitait arriver à des objectifs de réduction chiffrés, d'autre part les Etats-Unis, qui exigeaient la reconnaissance d'instruments économiques. Ces permis négociables leur permettraient d'acheter des «droits à polluer» auprès de pays qui émettraient moins de gaz à effet de serre qu'autorisés. Le protocole, rédigé et accepté, est maintenant soumis à signature des Etats parties à la convention. Les Etats-Unis ont d'ores et déjà fait dépendre leur signature de l'implication des pays en développement les plus importants dans le processus de réduction des émissions.

\section{Position suisse}

La Suisse prend le problème des changements climatiques très au sérieux. Par les mesures déjà prises en matière de politique climatique, elle avait la position la plus crédible pour négocier des objectifs de réduction de $\mathrm{CO}_{2}$. Grâce à un éventail de mesures, elle compte réduire de 10\% (par rapport au niveau de 1990) ses émissions de $\mathrm{CO}_{2}$ jusqu'à l'an 2000. Les politiques en matière environnementale, énergétique (Energie 2000), des transports et des finances comportent toutes un volet de protection du climat. L'instrument le plus ambitieux est certainement le projet de loi sur le $\mathrm{CO}_{2}$.

Les effets de la politique climatique suisse ont été évalués pour les années 1990 à 1995. Cette étude ${ }^{20}$ démontre que les émissions de $\mathrm{CO}_{2}$ sont restées stables et que la Suisse devrait réussir à les stabiliser en l'an 2000 à leur niveau de 1990.

Forte de ces acquis, la Suisse a défendu une position ambitieuse lors de la $\mathrm{COP}-3^{21}$. Lors d'une conférence de presse, elle a défendu une série de propositions devant figurer dans le protocole sur le climat:

20. Office fédéral de l'environnement, des forêts et du paysage, UN Frame Convention on Climate Change - Second National Communication of Switzerland 1997 - Greenhouse Inventory 1995, Bern, Swiss Confederation, April 1997.

21. OFEFP, Communiqué de presse, Berne, 11 novembre 1997, «La Suisse souhaite un protocole contraignant». 
- Principe de prévention: en dépit de certaines incertitudes scientifiques, des politiques et des mesures doivent être adoptées pour réduire les émissions de gaz à effet de serre.

- Principe du pollueur payeur: les pays produisant le plus d'émissions par habitant devraient faire les plus grands efforts de réduction.

- Le protocole devrait contenir les principes suivants:

- une réduction importante des émissions des trois gaz à effet de serre que sont le gaz carbonique, le méthane et le protoxyde d'azote;

- conformément au principe du pollueur payeur, des obligations variables selon les pays;

- des politiques et des mesures contraignantes visant à une réduction globale de $10 \%$ des émissions, coordonnées à l'échelle internationale pour les pays énumérés dans l'annexe I de la convention (pays industrialisés et pays d'Europe centrale et orientale).

D'autre part, la Suisse s'engage en faveur de l'introduction de la Joint Implementation.

\section{PROJET DE LOI SUR LE CO2}

JNE NOUvelle Lol sur la réduction des émissions de $\mathrm{CO}_{2}$ est en discussion devant le Parlement (sessions 1997 et printemps 1998). Ce projet de loi, élément central de la politique climatique suisse, vise à réduire les émissions de $\mathrm{CO}_{2}$ de $10 \%$ d'ici à 2010 par rapport à leur niveau de 1990. La réduction envisagée est de $5 \%$ pour les carburants et de $15 \%$ pour les combustibles.

Ces objectifs tiennent compte des politiques et mesures déjà adoptées, ainsi que celles prévues, pour réduire les émissions de $\mathrm{CO}_{2}$ dans les secteurs de l'énergie, des transports et de l'agriculture. La loi cherche également à favoriser les engagements volontaires et contractuels des milieux économiques. Une évaluation sera faite en 2004 et si le but de réduction n'est pas atteint par ces moyens, le projet de loi prévoit l'introduction d'une taxe sur le $\mathrm{CO}_{2}$.

A noter que la Suisse est, pour l'instant, le seul pays dont un projet de loi sur le $\mathrm{CO}_{2}$ soit en discussion devant le Parlement.

Source: "Message relatif à la loi fédérale sur la réduction des émissions de $\mathrm{CO}_{2}$ du 17 mars 1997》, in Feuille fédérale, vol. III, 3 juin 1997 (message 97.030).

Dans l'ensemble la Suisse est satisfaite des résultats obtenus à Kyoto, principalement des objectifs de réduction acceptés par les pays industrialisés. Selon elle, l'objectif défini dans le protocole correspond à celui du projet de loi. Le principe de sa ratification est déjà acquis.

Les organisations d'environnement présentes à la conférence de Kyoto ont fait savoir leur déception quant au contenu de l'accord auquel sont parvenus les gouvernements. Le WWF Suisse ${ }^{22}$ n'hésite pas à parler de véritable panier percé. Et de constater que cet accord est peu convaincant, truffé de clauses qui permettront aux nations pollueuses de continuer à relâcher des gaz à effet de serre dans

22. WWF Suisse, Communiqué de presse, 11 décembre 1997, «La Convention sur les changements climatiques signée à Kyoto est un véritable panier percé». 
l'atmosphère. Le WWF Suisse regrette «que le traité paraphé à Kyoto ne permette pas d'éloigner la menace d'un changement climatique car les acteurs, en particulier les Etats-Unis et le Japon, ont refusé de définir des objectifs réalistes pour réduire les émissions polluantes $(. .$.$) ».$

\section{CHRONOLOGIE DES PRINCIPALES ÉTAPES DE LA MISE EN GEUVRE DE LA CONVENTION SUR LE CLIMAT}

Décembre 1990

Publication du premier rapport d'évaluation du Groupe d'experts intergouvernemental sur l'évolution du climat (GIEC)

Juin 1992

Conférence des Nations Unies sur l'environnement et le développement, Rio de Janeiro

Signature par 155 pays de la Convention sur les changements climatiques

Ca ASTM 1993, pp. 64-66 (présentation de la convention)

21 mars $1994 \quad$ Entrée en vigueur de la convention

ASTM 1994, pp. 65-66 (premières ratifications de la convention et présentation Energie 2000)

21 septembre 1994 Entrée en vigueur de la Convention en Suisse

ASTM 1995, pp. 64-66 (ratification de la convention par la Suisse et Energie 2000/taxe $\mathrm{CO}_{2}$ )

Mars-avril $1995 \quad 1^{\text {re }}$ Conférence des parties (COP-1), Berlin

Mandat de Berlin: début des négociations concernant un protocole sur la réduction des émissions de gaz à effet de serre

ASTM 1996, pp. 51-57 (Conférence de Berlin, descriptif de la situation, taxe sur le $\mathrm{CO}_{2}$ )

Décembre $1995 \quad$ Publication du deuxième rapport du GIEC

Juillet 1996

$2^{\mathrm{e}}$ Conférence des parties (COP-2), Genève

Décision suisse de participer à la phase pilote de la Joint Implementation

$凹$ ASTM 1997, pp. 59-63 (rapport du GIEC, $2^{\circ}$ Conférence des parties)

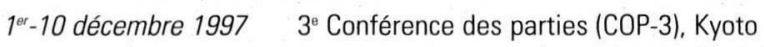

Signature du protocole par les parties à la convention

@ ASTM 1998

\section{SOURCES}

Office fédéral de l'environnement, des forêts et du paysage, UN Frame Convention on Climate Change - Second National Communication of Switzerland 1997 - Greenhouse Inventory 1995, Bern, Swiss Confederation, April 1997.

Office fédéral de l'environnement, des forêts et du paysage, Le climat en danger - Faits et perspectives concernant l'effet de serre, Berne, OFEFP, septembre 1997.

Office fédéral de l'environnement, des forêts et du paysage, Communiqué de presse, Berne, 11 novembre 1997, «La Suisse souhaite un protocole contraignant».

United Nations Environment Programme, Climate Change Information Kit, UNEP, January 1997.

Climate Change Secretariat, Press Kit - Kyoto 1997, October 1997.

Fritz Gassmann, Effet de serre - Modèles et réalités, collection Précis de l'environnement, Genève, Ed. Georg et SPE, 1996.

Le Monde diplomatique, «La survie planétaire marchandisée», janvier 1998.

\section{SITES INTERNET}

Site de la Convention sur les changements climatiques: http://www. unfccc. de

Site du PNUE consacré aux conventions internationales de protection de l'environnement: http://www. unep. ch/iuc. html

Site de l'Office fédéral de l'environnement, des forêts et du paysage: http://www. admin. ch/buwal/ 